\title{
A screening system for carbon sources enhancing $\beta$ - $N$-acetylglucosaminidase formation in Hypocrea atroviridis (Trichoderma atroviride)
}

\author{
Verena Seidl, Irina S. Druzhinina and Christian P. Kubicek
}

Correspondence

Verena Seidl

vseidl@mail.zserv.tuwien.ac.at

Received 3 February 2006

Revised 15 March 2006

Accepted 29 March 2006
Research Area Gene Technology and Applied Biochemistry, Institute of Chemical Engineering, TU Vienna, Getreidemarkt 9/166-5, A-1060 Vienna, Austria

To identify carbon sources that trigger $\beta$ - $N$-acetylglucosaminidase (NAGase) formation in Hypocrea atroviridis (anamorph Trichoderma atroviride), a screening system was designed that consists of a combination of Biolog Phenotype MicroArray plates, which contain 95 different carbon sources, and specific enzyme activity measurements using a chromogenic substrate. The results revealed growth-dependent kinetics of NAGase formation and it was shown that NAGase activities were enhanced on carbon sources sharing certain structural properties, especially on $\alpha$-glucans (e.g. glycogen, dextrin and maltotriose) and oligosaccharides containing galactose. Enzyme activities were assessed in the wild-type and a $H$. atroviridis $\Delta$ nag 1 strain to investigate the influence of the two NAGases, Nag1 and Nag2, on total NAGase activity. Reduction of NAGase levels in the $\Delta$ nag 1 strain in comparison to the wild-type was strongly carbon-source and growth-phase dependent, indicating the distinct physiological roles of the two proteins. The transcript abundance of nag 1 and nag 2 was increased on carbon sources with elevated NAGase activity, indicating transcriptional regulation of these genes. The screening method for the identification of carbon sources that induce enzymes or a gene of interest, as presented in this paper, can be adapted for other purposes if appropriate enzyme or reporter assays are available.

\section{INTRODUCTION}

Some species of the soil fungus Hypocrea (anamorph Trichoderma), e.g. Hypocrea atroviridis (Trichoderma atroviride) (Dodd et al., 2003), Hypocrea lixii (Trichoderma harzianum), Hypocrea virens (Trichoderma virens) and Trichoderma asperellum, are potent mycoparasites against several plant-pathogenic fungi, and lysis of the host cell wall has been demonstrated to be an important step in the mycoparasitic attack (Benítez et al., 2004; Chet et al., 1998; Howell, 2003; Kubicek et al., 2001). Consequently, with chitin being a major cell wall component of plant pathogens like Rhizoctonia solani, Botrytis cinerea and Sclerotinia sclerotiorum, several chitinolytic genes, encoding chitinases (EC 3.2.1.14) and $\beta$ - $N$-acetylglucosaminidases (NAGases; EC 3.2.1.52), have been cloned from Hypocreal Trichoderma spp. (Carsolio et al., 1994; Draborg et al., 1995; Garcia et al., 1994; Hayes et al., 1994; Kim et al., 2002; Peterbauer et al., 1996; Seidl et al., 2005; Viterbo et al., 2001, 2002) and for some of them the encoded protein also has been characterized (Boer et al., 2004; de la Cruz et al., 1992; Hoell et al., 2005). The regulation of expression of NAGases and

Abbreviations: NAGase, $\beta$ - $N$-acetylglucosaminidase; PM, Phenotype MicroArray; S.A., specific activity.

The GenBank/EMBL/DDBJ accession number for the sequence reported in this paper is DQ364461. chitinases in Hypocrea/Trichoderma has so far, besides Trichoderma-host interaction assays, only been studied with respect to their upregulation during growth on colloidal chitin, chitin degradation products and fungal cell walls (Carsolio et al., 1994; de las Mercedes Dana et al., 2001; Kim et al., 2002; Mach et al., 1999; Ramot et al., 2004). Additionally, the influence of carbon and nitrogen starvation on the expression of chitinolytic genes has been investigated (de las Mercedes Dana et al., 2001; Donzelli \& Harman, 2001; Mach et al., 1999). Detailed studies of the Hypocrea jecorina (Trichoderma reesei) genome revealed that this species has 18 different genes encoding glycoside family 18 chitinases, but interestingly only 2 genes encoding NAGases (glycoside family 20) (Seidl et al., 2005). Similar numbers can be expected for other Hypocreal Trichoderma spp. and the corresponding two genes encoding the NAGases have already been cloned from mycoparasitic Hypocrea/Trichoderma spp., namely nag1 from $H$. atrovir$i d i s, t v$-nagl and $t v$-nag2 from $H$. virens, exc1 and exc2 from $H$. lixii, and exc1y and exc2y from T. asperellum. It has been shown that transcription of $H$. atroviridis nagl is induced by fungal cell walls and low molecular mass chitooligosaccharides (Mach et al., 1999). Brunner et al. (2003) reported that nag1 is essential for triggering chitinase gene expression.

Although some of the host cell walls (e.g. those from ascomycetes and basidiomycetes) contain chitin, it is not 
readily available for Hypocrea/Trichoderma because it is linked to proteins and other polymers (De Groot et al., 2005; Mahadevan \& Tatum, 1967; Schoffelmeer et al., 1999). This raises the question as to which types of carbon sources derived from fungal cell walls possibly also trigger NAGase and chitinase expression, and act as inducers for the formation of chitinolytic enzymes in Hypocrea/ Trichoderma.

To investigate this, we have extended the Biolog Phenotype MicroArray (PM) system (Bochner et al., 2001; Bochner, 2003) towards a high-throughput system for screening carbon sources for their ability to induce NAGases. This system consists of 96-well microtitre plates containing 95 different carbon sources, and has recently been adapted to investigate carbon source utilization by filamentous fungi as a means of strain characterization (Druzhinina et al., 2006; Tanzer et al., 2003). We used a combination of the PMs with specific enzyme activity measurements with a chromogenic substrate to identify carbon sources that trigger NAGase formation in $H$. atroviridis, and compared those data with the transcript patterns of nag1 and nag2 obtained with realtime RT-PCR. To study the influence of Nag1 and Nag2 on total NAGase activity, enzyme activity was assessed in the wild-type and a $H$. atroviridis $\Delta$ nag1 strain.

\section{METHODS}

Strains and cultivation conditions. $H$. atroviridis P1 (ATCC 74058), referred to as wild-type, was maintained on PDA (Difco). The amdS ${ }^{+}$nnag1 strain H. atroviridis P1ND1 (Brunner et al., 2003) was kept on a minimal medium containing acetamide as the sole nitrogen source (Seidl et al., 2004).

The medium described previously by Seidl et al. (2005) containing $50 \mathrm{mM}$ MES ( $\mathrm{pH} 6.6$ ) and $1 \%$ (w/v) carbon source was used throughout the experiments not involving PMs. Agar plates $(1.5 \%$, $\mathrm{w} / \mathrm{v})$ were covered with cellophane, inoculated with $6 \times 10^{6}$ spores and incubated in constant darkness at $25^{\circ} \mathrm{C}$. Mycelia were harvested after $24,30,40$ and $48 \mathrm{~h}$ with a spatula, immersed in liquid $\mathrm{N}_{2}$ and stored at $-80^{\circ} \mathrm{C}$.

Biolog PMs. Carbon-utilization patterns were investigated using FF MicroPlates (Biolog). The FF MicroPlate test panel comprises 95 wells with different carbon-containing compounds and one well with water. Nutrients and test reagents are prefilled and dried into the 96 wells of the microplate.

Inoculum was extracted from Trichoderma strains after conidial maturation (5-8 days) by rolling a sterile, wetted cotton swab over sporulating areas. Conidia were suspended in $16 \mathrm{ml}$ sterile Phytagel solution $[0 \cdot 25 \%(\mathrm{w} / \mathrm{v})$ Phytagel, $0.03 \%(\mathrm{v} / \mathrm{v})$ Tween 40$]$ in disposable borosilicate test tubes $(20 \times 150 \mathrm{~mm})$. Phytagel is an agar-substitute gelling agent produced from a bacterial fermentation composed of glucuronic acid, rhamnose and glucose. The suspension was agitated in a vortex mixer for about $5 \mathrm{~s}$, and additional inoculum added as required to adjust the optical density of the suspension to $75( \pm 2) \%$ transmission at $590 \mathrm{~nm}$ wavelength. Conidial suspension $(60 \mu \mathrm{l})$ was dispensed into each of the wells of a Biolog FF MicroPlate. Inoculated microplates were incubated in the darkness at $25^{\circ} \mathrm{C}$, and $\mathrm{OD}_{750}$ readings determined after $12,18,24,36,42,48,66$ and $72 \mathrm{~h}$ using a microplate reader (Biolog), which measures the turbidity and reflects mycelial production on the tested substrate. Analyses were repeated at least three times for each strain. Joining cluster analysis - complete linkage rule and Euclidean distance measure as described by Druzhinina et al. (2006) - was employed to differentiate carbon sources depending on their utilization by $H$. atroviridis $\mathrm{P} 1$.

Enzyme activity measurements in Biolog PMs. NAGase activity was measured by a modification of the method of Yagi et al. (1989), which is based on the release of p-nitrophenol from the respective arylchitosides. After incubation of the microplates at $25^{\circ} \mathrm{C}$ in constant darkness for 30 and $48 \mathrm{~h}, 20 \mu \mathrm{l} 50 \mathrm{mM}$ potassium phosphate buffer, pH 6.7, containing $300 \mu \mathrm{g}$ 4-nitrophenyl $\mathrm{N}$-acetyl$\beta$-D-glucosaminide $\mathrm{ml}^{-1}$, was added to each well. Microplates were incubated at $30^{\circ} \mathrm{C}$ with gentle agitation. After $10 \mathrm{~min}$, the reactions were terminated by the addition of $20 \mu \mathrm{l} 0 \cdot 4 \mathrm{M} \mathrm{Na}_{2} \mathrm{CO}_{3}$ to each well. The plates were then put on ice for $5 \mathrm{~min}$ with gentle agitation to ensure complete mixing of the stop solution in the wells. Thereafter, the $A_{400}$ was determined in a microplate reader (MR7000; Dynex). The formation of product was linear with time during the observation interval (optimization data not shown). Control measurements of enzyme activity were performed by omitting the substrate from the phosphate buffer. Preliminary experiments proved that this yielded more reliable results than adding the $\mathrm{Na}_{2} \mathrm{CO}_{3}$ solution at $t=0$. Two independent assays, with a minimum of three separate plates for each reaction, were carried out.

Two sets of mean values were calculated from the $A_{400}$ values obtained in reactions with the substrate and from incubations without the substrate. For each carbon source the mean value of the control was then subtracted from the mean value of the enzymic measurement. In this way calculated enzymic activities, divided by the amount of biomass (expressed as $\mathrm{OD}_{750}$ units) formed at the corresponding time point, result in specific activities (S.A.s), given as arbitrary units. Outliers of enzyme activities were defined as values that were higher/ lower than the mean of the residual values \pm twofold SD. Basic statistical evaluations of data were performed using the STATISTICA 6.1 (StatSoft) software package.

RNA isolation. Total RNA was extracted as described by Chomczynski \& Sacchi (1987). Mycelia were disrupted using a bead mill homogenization method described by Griffith et al. (2000), with the FastPrep F120 (Qbiogene).

Cloning and sequencing of a nag2 orthologue from $\boldsymbol{H}$. atroviridis. The primers nag2-fw (5'-GCACGCTCTTCATTGACCAG-3') and nag2-rv (5'-CACAGTCATGCACATCAACCTG-3') were designed from conserved regions of $H$. lixii exc2 (GenBank accession no. S80070) for amplifying a $1.8 \mathrm{~kb}$ fragment of $H$. atroviridis nag2. The resulting sequence of the cloned DNA was submitted to GenBank (accession no. DQ364461).

Transcript analysis of nag1 and nag2 by real-time RT-PCR. RNA was treated with DNase I (Fermentas), purified with the RNeasy MinElute Cleanup kit (Qiagen) and reverse transcribed using the RevertAid $\mathrm{H}$ minus first strand cDNA synthesis kit (Fermentas) and the oligo $(\mathrm{dT})_{18}$ primer.

For real-time RT-PCR experiments a $130 \mathrm{bp}$ fragment of nag1 (GenBank accession no. S83231) was amplified with the primers nag1RT-fw ( $5^{\prime}$-GAACTGGAGGCTCATCTAC-3') and nag1RT-rv (5' GATGATGTTGTCCATGTTG-3'), and a 146 bp fragment of nag2 with the primers nag2RT-fw ( $5^{\prime}$-TGCGACCCGACCAAGAACTG- $3^{\prime}$ ) and nag2RT-rv (5'-CAGATGATGGTGTCGAGGCTG-3'). tef1 (encoding elongation factor $1 \alpha$, GenBank accession no. AF456892) was used as a reference gene, and a $100 \mathrm{bp}$ fragment was amplified with the primers tefRe-fw (5'-TACTGGTGAGTTCGAGGCTG-3') and tefRerv (5'-GATGGCAACGATGAGCTG-3').

Real-time PCR amplification was carried out with the iQ 5 real-time PCR detection system (Bio-Rad) in a $25 \mu$ reaction containing $12 \cdot 5 \mu \mathrm{l}$ 
iQ SYBR Green Supermix (Bio-Rad), each primer at a concentration of $250 \mathrm{nM}$ and sample corresponding to an initial concentration of $0.5 \mu \mathrm{g}$ total RNA. Amplification was carried out with the following PCR programme: initial denaturation for $3 \mathrm{~min}$ at $95^{\circ} \mathrm{C}$, followed by 40 cycles consisting of $95^{\circ} \mathrm{C}$ for $15 \mathrm{~s}, 52^{\circ} \mathrm{C}($ nag 1$), 58 \cdot 7^{\circ} \mathrm{C}$ (nag2) or $54^{\circ} \mathrm{C}$ (tefl), for $20 \mathrm{~s}$, and $72^{\circ} \mathrm{C}$ for $20 \mathrm{~s}$. Successful amplification was verified by determination of the melting temperature and by agarose gel electrophoresis. For each gene a series of dilutions were used for two different samples to assess the efficiency of the PCR. Two independent experiments were carried out and PCR reactions were performed in triplicates.

To ensure the absence of genomic DNA, control samples were subjected to the same procedure as described above, but no reverse transcriptase was added, and PCR reactions without template were set up to rule out contamination of other PCR components.

The results of the real-time RT-PCR were analysed with the iQ 5 optical system software (Bio-Rad). Using the PCR base line subtracted mode, the threshold cycle was calculated for all samples and the amplification efficiency for each gene was determined. To compare different samples, the threshold cycles for nag1 and nag2 were corrected with a factor for the tef1 amplification, as described by Reithner et al. (2005). The transcript value on glucose $(24 \mathrm{~h})$ was arbitrarily set to 1 and all other values given as multiples (fold induction) of it.

\section{RESULTS}

\section{Carbon source utilization profile of $\boldsymbol{H}$. atroviridis P1}

Prior to enzymic assays we examined the growth of $H$. atroviridis wild-type on 95 carbon sources under the conditions of the Biolog PMs. Detailed analysis of all growth curves (data not shown) led us to conclude that the time points 36,42 and $48 \mathrm{~h}$ correspond to the phase of linear (active) growth on the majority of carbon sources. This observation is consistent with previous results for $H$. jecorina (Druzhinina et al., 2006). We applied joining cluster analysis to $\mathrm{OD}_{750}$ values from these time points only, to detect possible groupings of carbon sources depending on the respective growth kinetics. Data for previous (germination) and subsequent (growth saturation and sporulation) phases were used as a reference when needed. The general carbon-source utilization profiles for $H$. atroviridis are represented by four distinct clusters (Fig. 1). Cluster I contained the best utilizable carbon sources for this species, which led to the fastest growth and in most cases resulted in termination after $48 \mathrm{~h}$. It comprised mainly monosaccharides and polyols, and also $\gamma$-amino-butyric acid, which is reported to be the best carbon source for $H$. jecorina (Druzhinina et al., 2006). Additionally, it was conspicuous that $N$-acetyl-D-glucosamine belonged to cluster I, while neither other hexosamines nor D-glucosamine promoted fast growth for $H$. atroviridis. Cluster II contained again mostly monosaccharides, and also some oligosaccharides and arylglucosides. On these carbon sources $H$. atroviridis exhibited a slower increase in biomass formation compared to cluster I sources, which was constant during the whole time-course of the experiment $(72 \mathrm{~h})$. Cluster III comprised carbon sources on which biomass formation started with a considerable delay (between 42 and $48 \mathrm{~h}$ ) and contained predominantly disaccharides and oligosaccharides, arylglucosides and L-amino acids. Cluster IV contained several L-amino acids, peptides, biogenic and heterocyclic amines, some TCA-cycle intermediates, and aliphatic organic acids, which promoted only very slow growth at $48 \mathrm{~h}$. Weak and delayed biomass formation was detectable on some of those carbon sources, but the majority of them led to no growth at all.

\section{Carbon sources inducing NAGase activity}

We examined NAGase activity in $H$. atroviridis after 30 and $48 \mathrm{~h}$ directly in the Biolog PMs, which has the advantage that the measurement includes both the enzyme secreted into the medium and that bound to the fungal cell wall. Results of the NAGase activity measurements after $30 \mathrm{~h}$ are shown in Fig. 2(a). The obtained values displayed low variance, indicating reproducible enzyme activity measurements.

The results showed a statistically significant correlation between NAGase activity and biomass formation $(r=0 \cdot 60$, $P<0 \cdot 05$; Fig. 2a) after $30 \mathrm{~h}$. The growth rate influenced the level of NAGase S.A.s, with faster growth rates leading to a statistically significant increase in NAGase S.A.s $(r=0.42, P<0.05$; Fig. 2b). NAGases were formed on most carbon sources, but only a minor number yielded elevated S.A.s (see below).

With regard to enzyme activity, we defined two grades of induction: weakly inducing carbon sources, which produced an increase in S.A. of $150-200 \%$ of the calculated mean of all carbon sources for a given time point, and moderately inducing carbon sources, which produced S.A.s higher than $200 \%$ of the mean. $\mathrm{N}$-acetyl-D-glucosamine was the only carbon source that caused strong NAGase induction (cf. Fig. 2a, b), and therefore it was omitted from the calculations of mean NAGase values.

After $30 \mathrm{~h}$ of growth, increased NAGase S.A.s were found on carbon sources that mainly belonged to the clusters I and II based on the respective growth kinetics. After $48 \mathrm{~h}$ of growth there was a marked shift of the affiliation of carbon sources causing increased NAGase S.A.s from clusters I/II to cluster II/III (Fig. 3). This correlates well with the fact that cluster III contains those carbon sources where active growth and biomass formation starts at $48 \mathrm{~h}$.

In addition to the strong inducer $\mathrm{N}$-acetyl-D-glucosamine, which is known to induce NAGase formation even at concentrations as low as $1 \mathrm{mM}$ (Mach et al., 1999), the following carbon sources also resulted in elevated NAGase activity: the $\alpha 1 \rightarrow 4$ linked glucans/glucosides glycogen, dextrin, and maltotriose, $\alpha$ - and $\beta$-cyclodextrin and maltose, the $\beta$-glucosides cellobiose and $\beta$-methylglucoside, the $\alpha$-glucosides palatinose, turanose, salicin and arbutin, the sugar acids 2-keto-D-gluconate and D-glucuronate, the monosaccharides D-ribose, L- and D-arabinose, D-mannose, 


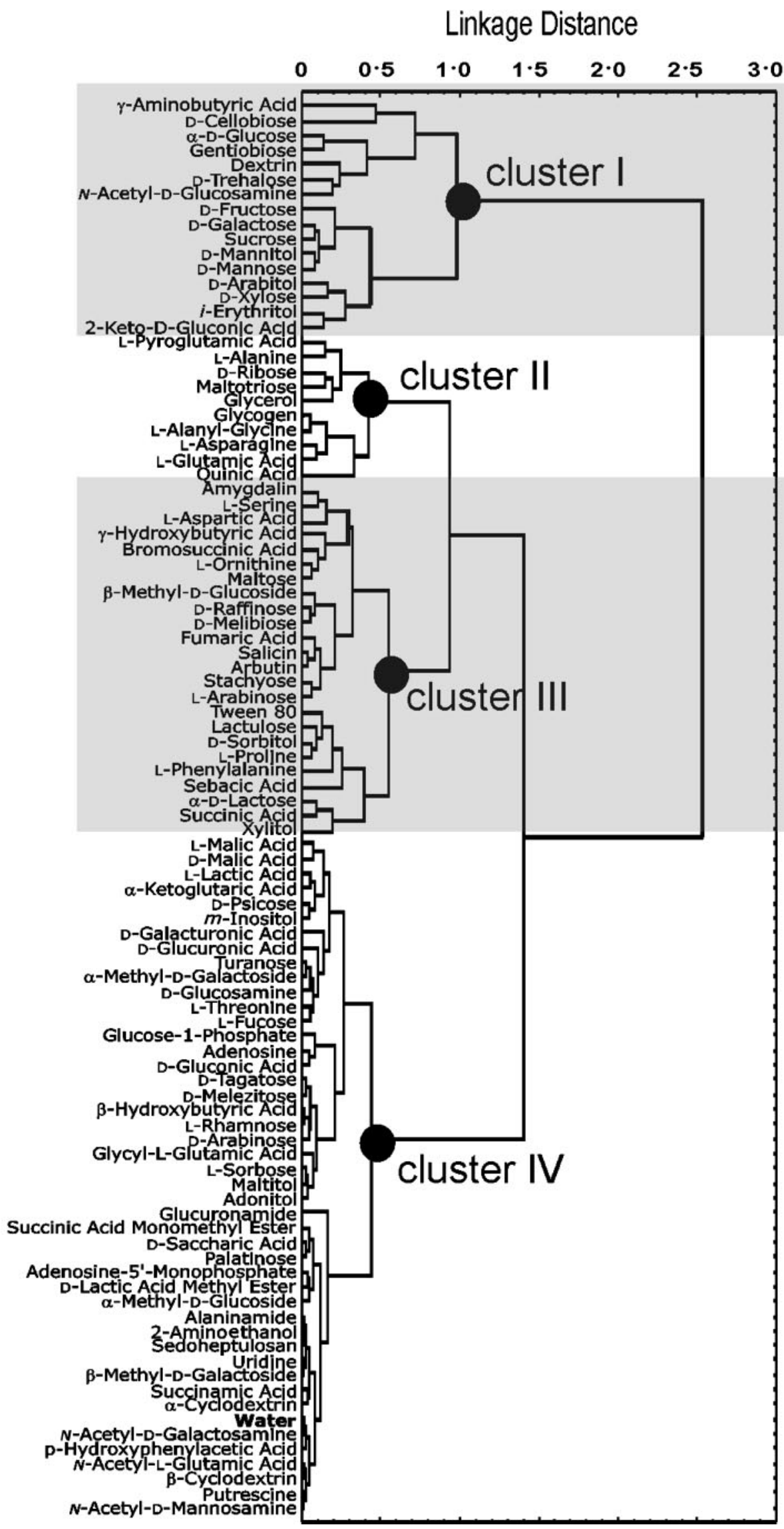



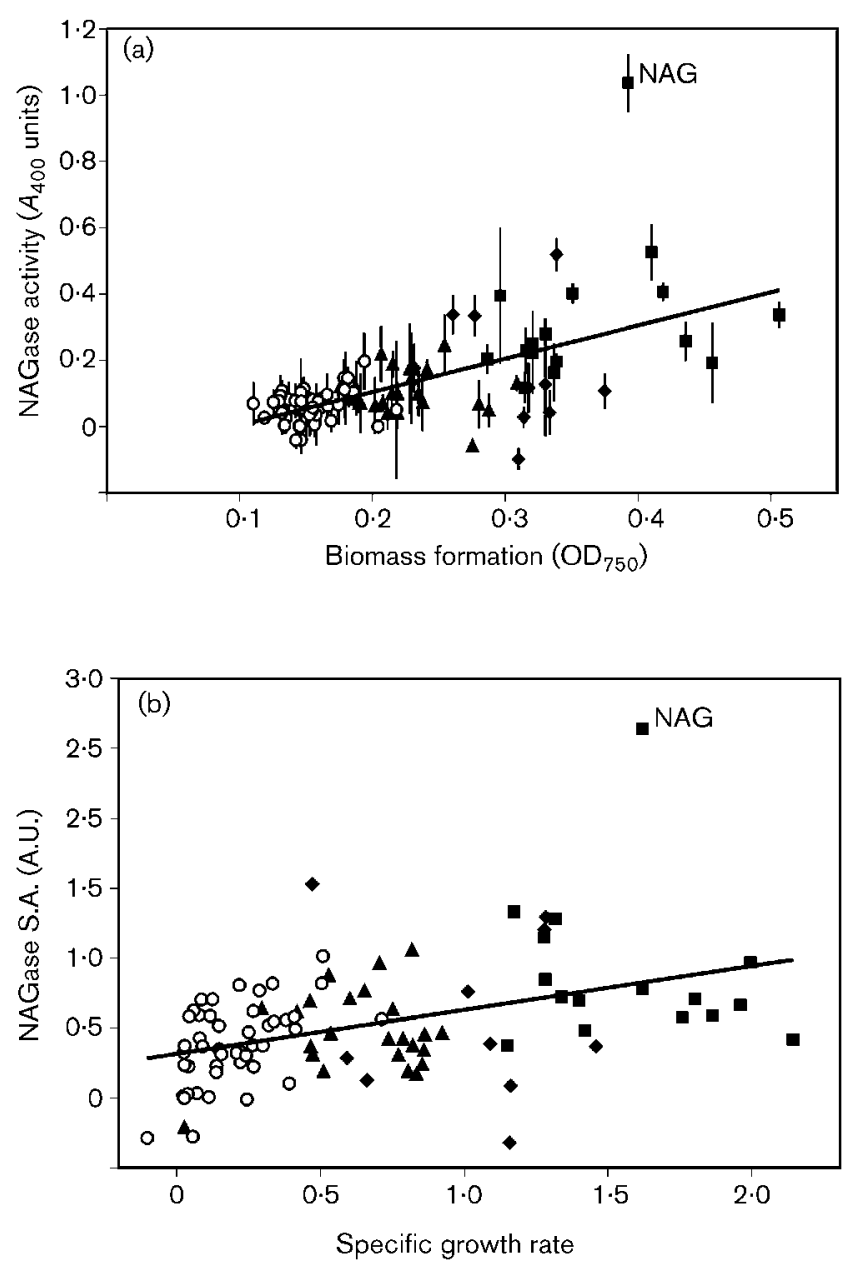

Fig. 2. (a) Correlation of NAGase activity with biomass after $30 \mathrm{~h}$ of growth. Vertical bars indicate the SDs for the NAGase activity measurements. (b) Influence of the specific growth rate (the growth rate between 24 and $36 \mathrm{~h}$ divided by the biomass at $24 \mathrm{~h}$ ) on NAGase S.A.s after $30 \mathrm{~h}$ of growth. $\mathbf{0}$, Cluster I; $\checkmark$, cluster II; $\boldsymbol{\Delta}$, cluster III; $O$, cluster IV; $\mathbf{D A G}, N$-acetyl-Dglucosamine. The diagonal line is the trendline. A.U., arbitrary units.

D-fructose, D-sorbitol (which is also a constituent of Tween 80 ), psicose, adonitol, $m$-inositol, and Tween 80 (polyoxyethylensorbitan monooleate) and $\beta$-hydroxybutyric acid. Additionally, it was conspicuous that NAGase activity was enhanced on the D-galactose containing carbohydrates D-melibiose, D-raffinose and stachyose, lactulose, $\alpha$-Dlactose, $N$-acetyl-D-galactosamine and $\alpha$-methylgalactoside, and also the $\mathrm{D}$-galactose derivates fucose and $\mathrm{D}$-galacturonic acid. The NAGase S.A.s that could be found in the well solely containing water can be explained by the fact that the Phytagel spore carrier is a heteropolysaccharide composed of glucuronic acid, rhamnose (6-deoxymannose) and glucose.

\section{Carbon sources inducing NAGase activity in a $\Delta$ nag1 strain}

For the above described results we measured total NAGase S.A., which in fact is a mixture of the activity of the two NAGases Nag1 and Nag2. To identify whether the Nag1 and the remaining NAGase activity are coordinately or differentially regulated by inducing substances, enzyme activity measurements were carried out with a $H$. atroviridis $\Delta$ nag1 strain (Brunner et al., 2003). We did not find significant differences when the phenotype profile of the $\Delta$ nagl and the wild-type strain were compared. With respect to enzyme activities, the $\Delta$ nagl strain showed a strong reduction of NAGase activity on most carbon sources compared to the wild-type. This demonstrated that Nag1 was mainly responsible for the total NAGase S.A.s in the wild-type. However, the effect was still strongly carbon-source dependent as can be seen in Fig. 4.

The main role of Nag1 during hyphal growth became apparent when the reduction of NAGase activity was compared for different growth clusters. After $30 \mathrm{~h}$, clusters I and II, promoting fast growth and high biomass yield, had a mean reduction of $65 \%$ of NAGase activity, while the reduction was only $38 \%$ in cluster III and $16 \%$ in cluster IV.

The reduction of NAGase activity was even more pronounced after $48 \mathrm{~h}$ of growth: a mean reduction of $64 \%$ of NAGase activity for carbon sources of cluster I, $56 \%$ for cluster II, $76 \%$ for cluster III and $55 \%$ for cluster IV.

\section{Transcript analysis by real-time RT-PCR of nag 1 and nag2}

In order to test whether the data obtained by enzyme measurements actually reflect the expression of the nag1 and nag2 genes, we scaled up the incubation experiments to obtain enough mycelia for the extraction of RNA. Preliminary experiments with submerged cultivations (shake-flask cultures) showed that biomass formation was accompanied by early sporulation on carbon sources that provided slow growth of $H$. atroviridis, whereas on agar plates the fungus was growing slowly, but did not sporulate during growth. Consequently, cultivations on agar plates containing the respective carbon sources and covered with cellophane were chosen to obtain mycelial biomass for real-time RT-PCR analysis of nag1 and nag2 transcript formation. A representative set of carbon sources that displayed elevated NAGase S.A.s, namely dextrin, glycogen, maltotriose, D-melibiose, D-raffinose, $\beta$-methylglucoside and $m$-inositol, were chosen for these experiments.

The results (Fig. 5a, b) demonstrate that the NAGase activity described above is in good accordance with the

Fig. 1. Utilization of carbon sources by $H$. atroviridis $P 1$. Joining cluster analysis was applied to mycelial growth values $\left(\mathrm{OD}_{750}\right.$ units) at 36,42 and $48 \mathrm{~h}$, which correspond to the linear growth phase on the majority of carbon sources. Branching points of clusters. 


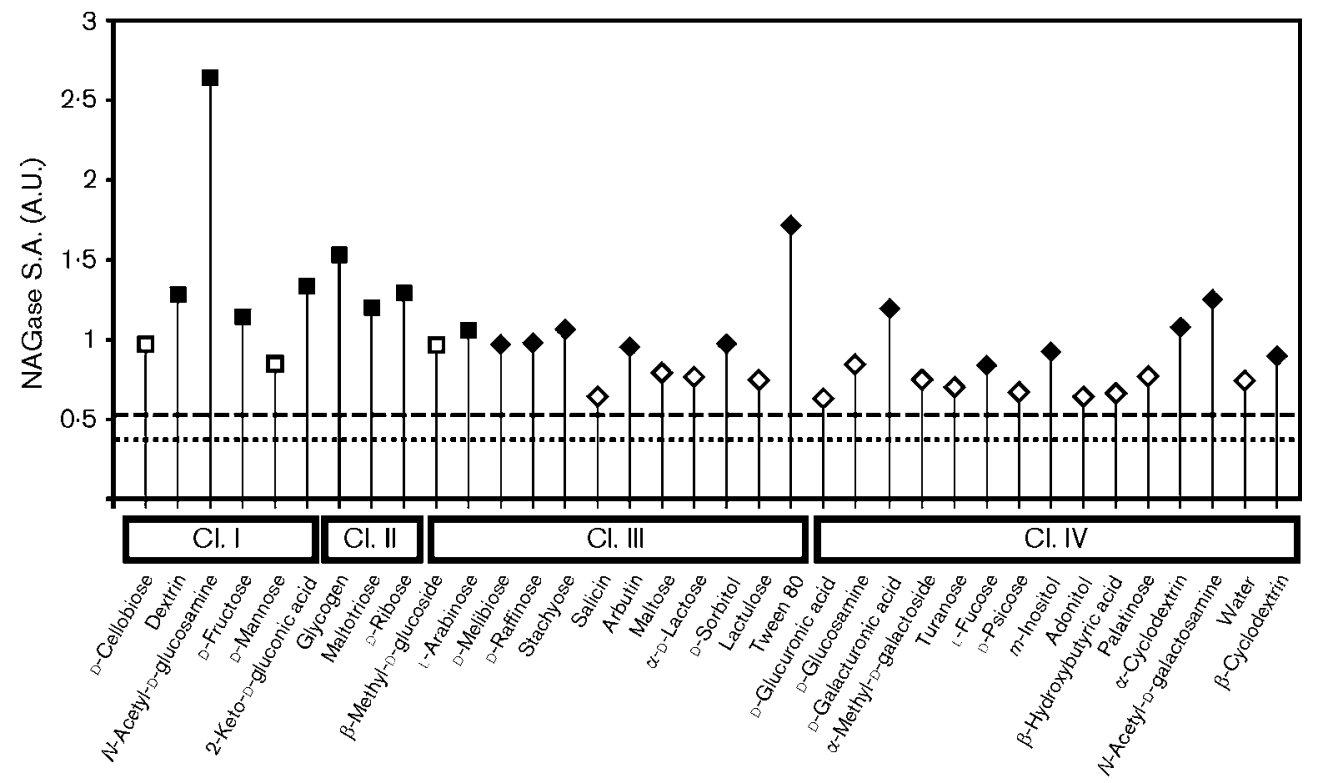

Fig. 3. NAGase S.A. in $H$. atroviridis on carbon sources from different growth clusters. Values above $200 \%$ of the mean are indicated by $\square(30 \mathrm{~h})$ and $\diamond(48 \mathrm{~h})$, and values between 150 and $200 \%$ are indicated by $\square(30 \mathrm{~h})$ and $\diamond(48 \mathrm{~h})$. The dashed and dotted lines show the mean for all carbon sources at 30 and 48 h, respectively. Cl., cluster; A.U., arbitrary units.

respective transcript abundance of nag1 and nag2. Growth on carbon sources that caused elevated NAGase activity resulted in higher transcript formation than the negative controls glucose and glycerol, indicating that nag1 and nag2 are regulated at the transcriptional level. Increased nag1 transcript formation was statistically significant at 24 and $30 \mathrm{~h}$ (one-way ANOVA, $F(1,18)=6 \cdot 97, P=0 \cdot 02$ ). The abundance of the nag2 transcript (Fig. 5b) essentially reflected the relative abundances of the nag1 transcript. Thus, nag1 and nag2 transcription increased twofoldfourfold on 'inducing' carbon sources in comparison with glucose and glycerol, but the data also suggest that nag1 is more strongly regulated than nag2 on carbon sources that provide faster growth.

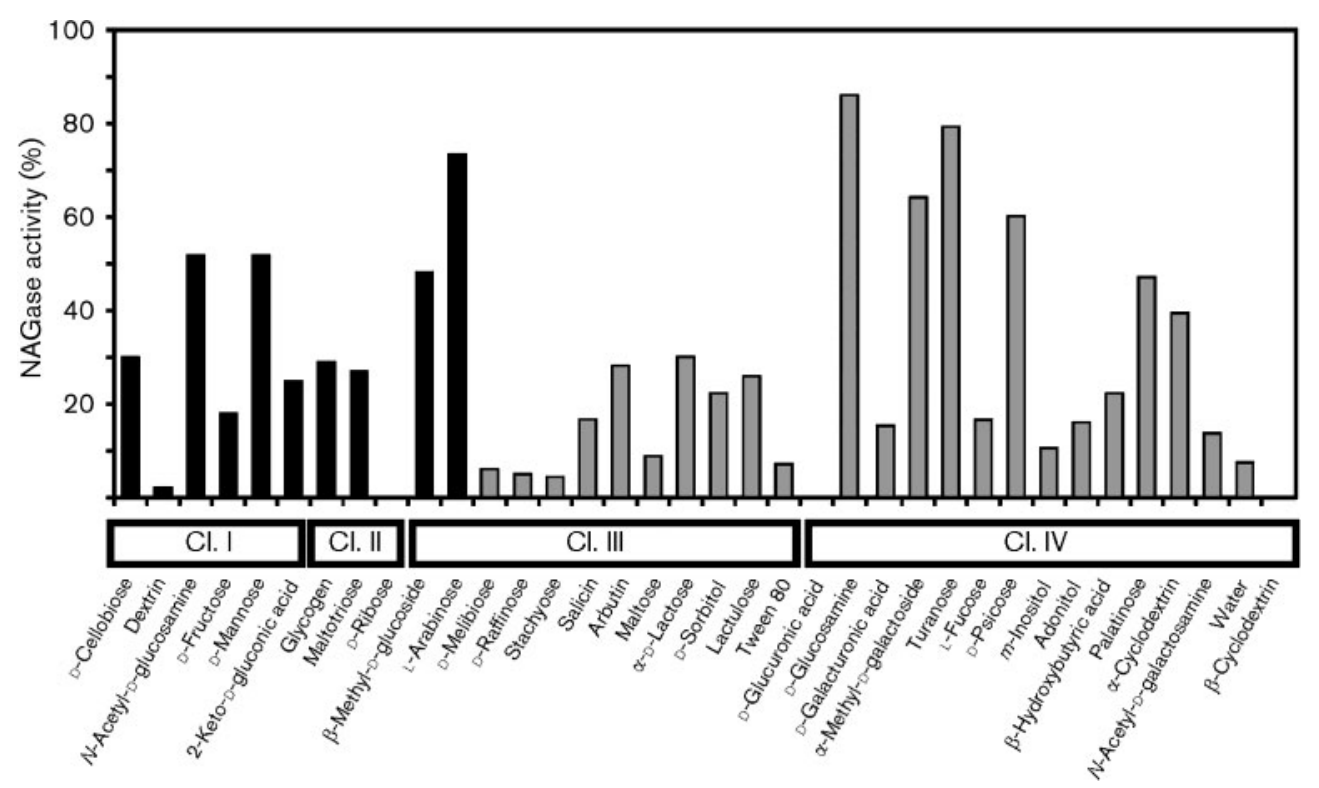

Fig. 4. Reduction of NAGase S.A.s in $H$. atroviridis $\Delta$ nag1. Values are given as a percentage in relation to NAGase S.A.s in the $H$. atroviridis wild-type (Fig. 3). Black bars, $30 \mathrm{~h}$; grey bars, $48 \mathrm{~h}$. 

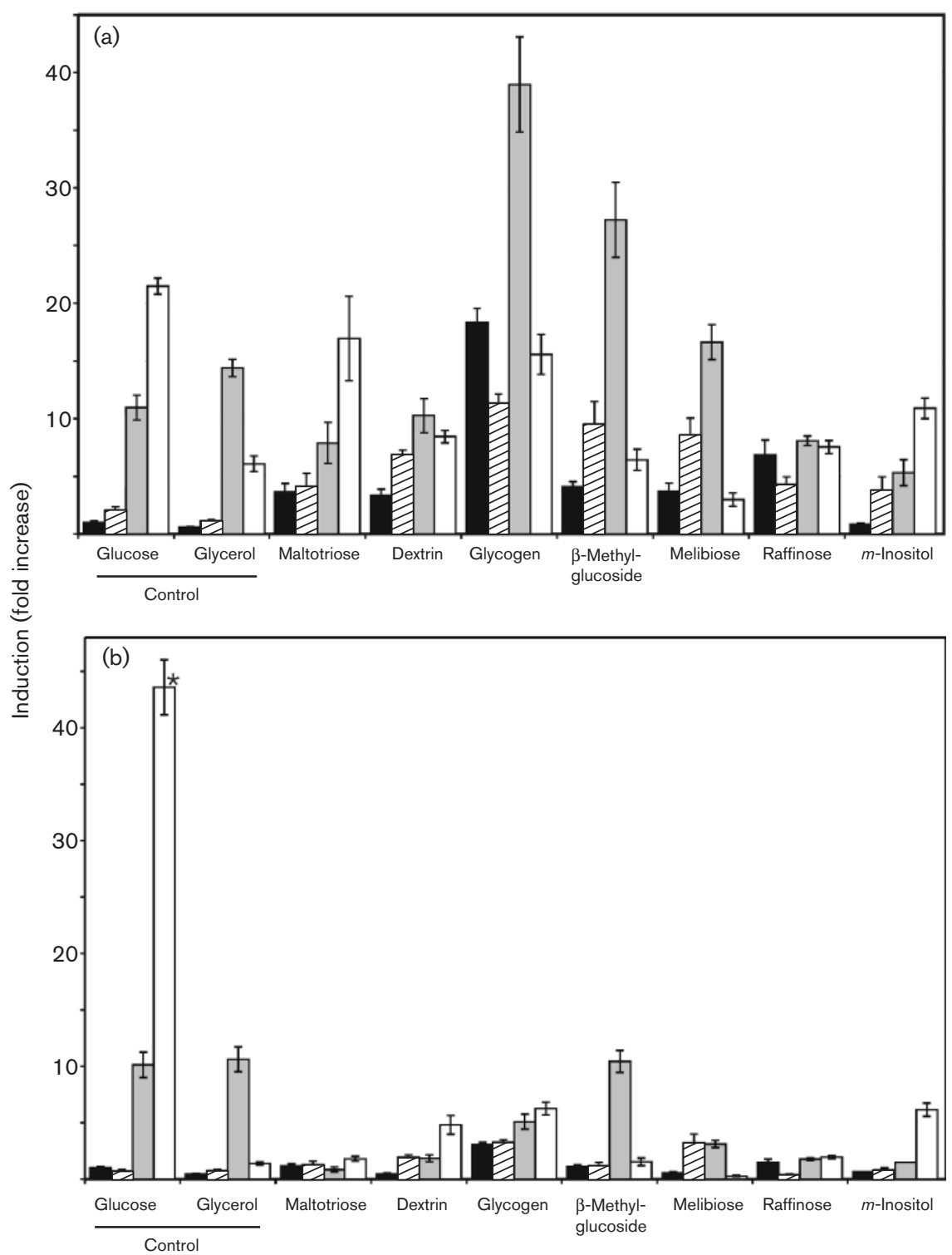

Fig. 5. Results of transcript analysis of nag1 and nag2 after growth for 24, 30, 40 and $48 \mathrm{~h}$ on selected carbon sources. The values given are ratios of (a) nag 1 and (b) nag2 transcript levels, normalized to tef1 as determined by real-time RT-PCR, and are shown as fold induction in relation to the respective values for glucose at $24 \mathrm{~h}$, which was set 1 . Black bars, $24 \mathrm{~h}$; hatched bars, $30 \mathrm{~h}$; grey bars, $40 \mathrm{~h}$; white bars, $48 \mathrm{~h}$; *, samples where conidiation could be observed.

\section{DISCUSSION}

In this study we analysed the stimulation of NAGase formation in $H$. atroviridis influenced by various carbon sources. A number of carbon sources that clearly enhanced NAGase activity were detected. They comprised $\alpha$-glucans, like glycogen, dextrin and maltotriose, and several oligosaccharides, particularly those containing D-galactose. Polysaccharides with the same type of glycosidic linkage are constituents of the cell wall of the majority ascomycetes (Latgé et al., 2005; Schoffelmeer et al., 1999; Tomazett et al., 2005), including plant pathogens (Wolski et al., 2005). Moreover, the formation of a 1,3- $\alpha$-glucanase has been shown to be part of the mycoparasitic response of $H$. lixii (Sanz et al., 2005). The stimulation of NAGase activity by $\alpha$-glucans and D-galactose containing oligosaccharides may thus be part of a mechanism by which $H$. atroviridis senses the presence of a host cell wall containing chitin. In fact, chitin is deeply imbedded within the fungal cell wall (Mahadevan \& Tatum, 1967) and not readily accessible without attack of the outer glucaneous layer. The availability of the respective oligosaccharides may signal that a cell wall degradation process has just been started.

As expected, $\mathrm{N}$-acetyl-D-glucosamine, which has already been reported to induce nagl expression in $H$. atroviridis and other Hypocreal Trichoderma spp. (Brunner et al., 2003; Mach et al., 1999; Peterbauer et al., 1996, 2002), was also the strongest soluble inducer of NAGase activity among all tested carbon sources in our experiments.

It is an important finding that NAGase activity was not enhanced at low growth rates. This indicates that the stimulatory effect of various carbon sources detected in this study is not caused by carbon-catabolite derepression at decreased growth rates (Ilyes et al., 2004). In fact, to date 
there is no evidence that either nag1 or nag2 would be subject to carbon-catabolite repression at all.

Comparison of NAGase formation in the $H$. atroviridis wild-type strain and the $\Delta$ nagl mutant showed that the reduction of NAGase activity varied greatly among the different carbon sources, and furthermore, the ratio was not constant but dependent on the growth phase. NAGase activity induced by D-glucosamine was almost completely maintained in the $H$. atroviridis $\Delta$ nag1 mutant, indicating that D-glucosamine mainly induces nag2. In the same strain, induction by $\mathrm{N}$-acetyl-D-glucosamine was reduced to about $50 \%$. Therefore, nag1 is mainly induced by $N$-acetyl-Dglucosamine, whereas nag2 is induced by both this carbon source and D-glucosamine. Interestingly, these two carbon sources are assimilated by $H$. atroviridis at different rates: $\mathrm{N}$-acetyl-D-glucosamine is utilized rapidly, whereas D-glucosamine provides only slow growth. This suggests that the poor utilization of D-glucosamine is most likely due to an inefficient uptake. In view of this, the fact that both $\mathrm{N}$-acetyl-D-glucosamine and D-glucosamine induce nag2 indicates that the induction is caused before the uptake into the cell, e.g. by a receptor-mediated mechanism, which deserves further investigation.

D-glucosamine was reported to cause stronger induction of residual NAGases in a strain deleted in the nag2 orthologue in T. asperellum than $\mathrm{N}$-acetyl-D-glucosamine (Ramot et al., 2004). Unfortunately, no comparison to the wild-type was given in that paper; therefore, the proportion of nag2 of total NAGase activity cannot be deduced. Although D-glucosamine also caused elevated NAGase activity in our experiments, the induction was only moderate in comparison with other carbon sources. This difference could be explicable by the fact that Ramot et al. (2004) used shake-flask cultures in their study, while we tested for NAGase activity in solid media. The influence of the cultivation method under otherwise similar conditions on gene expression has recently been the subject of several studies (Holker et al., 2004; te Biesebeke et al., 2005a, b). However, it should be noted that we also did not get high NAGase activities when $H$. atroviridis was grown directly on D-glucosamine in shakeflask cultures (data not shown) and, therefore, we consider it likely that the different inducibility of NAGases by $\mathrm{N}$-acetyl-D-glucosamine and D-glucosamine could be due to the interspecific variability between $H$. atroviridis and $T$. asperellum.

Disproportionately high levels of NAGase activity remained in the $\Delta$ nag1 mutant when it was grown on some compounds such as L-arabinose, turanose and D-psicose, indicating a preferential induction of nag 2 by these compounds. These findings show that Nag1 and Nag2 are not redundant but probably have different, specific functions in $\mathrm{H}$. atroviridis metabolism. Separate analysis of nag1 and nag2 transcription on selected carbon sources generally confirmed the induction deduced from measurement of enzyme activity, although the relative abundance of the nag2 transcript varied less strongly than was deduced from the differences in
NAGase activity between the wild-type and the $\Delta$ nagl mutant. Brunner et al. (2003) have shown that the presence of Nag1 is necessary for full induction of chitinase activity in $H$. atroviridis, and it is possible that it also influences the induction of nag2. However, other factors such as stability of the enzyme and proteolytic degradation may influence this process.

Multiple genes encoding NAGases are also present in all the fungi whose genome sequences are available today and which are not mycoparasites (e.g. Phanerochaete chrysosporium, Neurospora crassa, Magnaporthe grisea, Fusarium graminearum, B. cinerea, Aspergillus fumigatus, Aspergillus nidulans, Aspergillus oryzae). This implies that the physiological role of these enzymes is not exclusively connected with mycoparasitism. The positive correlation between NAGase activity and the growth rate in $H$. atroviridis, as found in this work, and its occurrence in the cell wall (Brunner et al., 2003; Ramot et al., 2004) suggests an involvement of these enzymes in cell wall turnover. This is consistent with previous results (Brunner et al., 2003) that the $\Delta$ nagl strain has a reduced rate of autolysis.

The screening system developed in this paper was based on a combination of PMs and an enzymic assay using a chromogenic substrate. It is a fast and reliable method to measure enzymic activities on a large set of carbon sources. Also, it can be adapted for enzyme activity measurements of a variety of extracellular and cell wall bound enzymes. By using appropriate promoter-fusion reporter systems, this method can be further used to monitor the expression of specific genes, even encoding intracellular enzymes. In fact, we have already tested one such system using the secreted Aspergillus niger glucose oxidase encoding goxA gene fused to the nag1 promoter (Mach et al., 1999), and the data obtained (V. Seidl, unpublished data) were generally concordant with those reported in this study. However, other highly sensitive and secretion-independent reporter systems, such as GFP (Larrainzar et al., 2005) or luciferase (Morgan et al., 2003), may prove to be even more effective in combination with the PM system. The rapidly growing number of fungal genome sequence databases is leading to an increase in the identification of genes for which orthologues in even closely related species do not exist (Dogra \& Breuil, 2004; O'Brian et al., 2003; Schmoll et al., 2004). Such findings direct the attention of researchers to novel, yet uncharacterized, enzymes with unknown substrate specificities and physiological functions. Even for proteins with defined enzymic activities knowledge about their physiological roles is often restricted to transcript analysis for a limited set of growth conditions. Having an array-type system available to screen carbon sources and/or growth conditions under which a novel gene is actually expressed would facilitate assigning functions to newly found genes and greatly increase the knowledge about their metabolic functions. In fact, the differences in regulation between nag1 and nag2 as shown in this work would probably have gone undetected without this tool. 


\section{ACKNOWLEDGEMENTS}

This study was supported by EU-funded TrichoEST project (QLK32002-02032). The authors thank Bernhard Seiboth for helpful suggestions and critically reading the manuscript.

\section{REFERENCES}

Benítez, T., Rincon, A. M., Limón, M. C. \& Codon, A. C. (2004). Biocontrol mechanisms of Trichoderma strains. Int Microbiol 7, 249-260.

Bochner, B. R. (2003). New technologies to assess genotypephenotype relationships. Nat Rev Genet 4, 309-314.

Bochner, B. R., Gadzinski, P. \& Panomitros, E. (2001). Phenotype MicroArrays for high-throughput phenotypic testing and assay of gene function. Genome Res 11, 1246-1255.

Boer, H., Munck, N., Natunen, J., Wohlfahrt, G., Soderlund, H., Renkonen, O. \& Koivula, A. (2004). Differential recognition of animal type $\beta 4$-galactosylated and $\alpha 3$-fucosylated chito-oligosaccharides by two family 18 chitinases from Trichoderma harzianum. Glycobiology 14, 1303-1313.

Brunner, K., Peterbauer, C. K., Mach, R. L., Lorito, M., Zeilinger, S. \& Kubicek, C. P. (2003). The Nag1 $N$-acetylglucosaminidase of Trichoderma atroviride is essential for chitinase induction by chitin and of major relevance to biocontrol. Curr Genet 14, 289-295.

Carsolio, C., Gutierrez, A., Jimenez, B., Van Montagu, M. \& HerreraEstrella, A. (1994). Characterization of ech-42, a Trichoderma harzianum endochitinase gene expressed during mycoparasitism. Proc Natl Acad Sci U S A 91, 10903-10907.

Chet, I., Benhamou, N. \& Haran, S. (1998). Mycoparasitism and lytic enzymes. In Trichoderma and Gliocladium Enzymes, Biological Control and Commercial Applications, pp. 153-172. Edited by G. E. Harman \& C. P. Kubicek. London: Taylor \& Francis.

Chomczynski, P. \& Sacchi, N. (1987). Single-step method of RNA isolation by acid guanidinium thiocyanate-phenol-chloroform extraction. Anal Biochem 162, 156-159.

De Groot, P. W., Ram, A. F. \& Klis, F. M. (2005). Features and functions of covalently linked proteins in fungal cell walls. Fungal Genet Biol 42, 657-675.

de la Cruz, J., Hidalgo-Gallego, A., Lora, J. M., Benítez, T., PintorToro, J. A. \& Llobell, A. (1992). Isolation and characterization of three chitinases from Trichoderma harzianum. Eur J Biochem 206, 859-867.

de las Mercedes Dana, M., Limón, M. C., Mejías, R., Mach, R. L., Benitez, T., Pintor-Toro, J. A. \& Kubicek, C. P. (2001). Regulation of chitinase 33 (chit33) gene expression in Trichoderma harzianum. Curr Genet 38, 335-342.

Dodd, S., Lieckfeldt, E. \& Samuels, G. J. (2003). Hypocrea atroviridis sp. nov., the teleomorph of Trichoderma atroviride. Mycologia 95, 27-40.

Dogra, N. \& Breuil, C. (2004). Suppressive subtractive hybridization and differential screening identified genes differentially expressed in yeast and mycelial forms of Ophiostoma piceae. FEMS Microbiol Lett 238, 175-181.

Donzelli, B. G. \& Harman, G. E. (2001). Interaction of ammonium, glucose, and chitin regulates the expression of cell wall-degrading enzymes in Trichoderma atroviride strain P1. Appl Environ Microbiol 67, 5643-5647.

Draborg, H., Kauppinen, S., Dalboge, H. \& Christgau, S. (1995). Molecular cloning and expression in S. cerevisiae of two exochitinases from Trichoderma harzianum. Biochem Mol Biol Int 36, 781-791.
Druzhinina, I., Schmoll, M., Seiboth, B. \& Kubicek, C. P. (2006). Global carbon utilization profiles of wild type, mutant and transformant strains of Hypocrea jecorina. Appl Environ Microbiol 72, 2126-2133.

Garcia, I., Lora, J. M., de la Cruz, J., Benítez, T., Llobell, A. \& PintorToro, J. A. (1994). Cloning and characterization of a chitinase (chit42) cDNA from the mycoparasitic fungus Trichoderma harzianum. Curr Genet 27, 83-89.

Griffiths, R. I., Whiteley, A. S., O'Donnell, A. G. \& Bailey, M. J. (2000). Rapid method for coextraction of DNA and RNA from natural environments for analysis of ribosomal DNA- and rRNA-based microbial community composition. Appl Environ Microbiol 66 5488-5491.

Hayes, C. K., Klemsdal, S., Lorito, M., Di Pietro, A., Peterbauer, C. Nakas, J. P., Tronsmo, A. \& Harman, G. E. (1994). Isolation and sequence of an endochitinase-encoding gene from a cDNA library of Trichoderma harzianum. Gene 138, 143-148.

Hoell, I. A., Klemsdal, S. S., Vaaje-Kolstad, G., Horn, S. J. \& Eijsink, V. G. (2005). Overexpression and characterization of a novel chitinase from Trichoderma atroviride strain P1. Biochim Biophys Acta 1748, 180-190.

Holker, U., Hofer, M. \& Lenz, J. (2004). Biotechnological advantages of laboratory-scale solid-state fermentation with fungi. Appl Microbiol Biotechnol 64, 175-186.

Howell, C. R. (2003). Mechanisms employed by Trichoderma spp. in the biological control of plant diseases: the history and evolution of current concepts. Plant Dis 87, 4-10.

llyes, H., Fekete, E., Karaffa, L., Fekete, E., Sandor, E., Szentirmai, A. \& Kubicek, C. P. (2004). CreA-mediated carbon catabolite repression of $\beta$-galactosidase formation in Aspergillus nidulans is growth rate dependent. FEMS Microbiol Lett 235, 147-151.

Kim, D. J., Baek, J. M., Uribe, P., Kenerley, C. M. \& Cook, D. R. (2002). Cloning and characterization of multiple glycosyl hydrolase genes from Trichoderma virens. Curr Genet 40, 374-384.

Kubicek, C. P., Mach, R. L., Peterbauer, C. K. \& Lorito, M. (2001). Trichoderma: from genes to biocontrol. J Plant Pathol 83, 11-23.

Larrainzar, E., O'Gara, F. \& Morrissey, J. P. (2005). Applications of autofluorescent proteins for in situ studies in microbial ecology. Annu Rev Microbiol 59, 257-277.

Latgé, J. P., Mouyna, I., Tekaia, F., Beauvais, A., Debeaupuis, J. P. \& Nierman, W. (2005). Specific molecular features in the organization and biosynthesis of the cell wall of Aspergillus fumigatus. Med Mycol 43, 15-22.

Mach, R. L., Peterbauer, C. K., Payer, K., Jaksits, S., Woo, S. L., Zeilinger, S., Kullnig, C. M., Lorito, M. \& Kubicek, C. P. (1999). Expression of two major chitinase genes of Trichoderma atroviride (T. harzianum P1) is triggered by different regulatory signals. Appl Environ Microbiol 65, 1858-1863.

Mahadevan, P. R. \& Tatum, E. L. (1967). Localization of structural polymers in the cell wall of Neurospora crassa. J Cell Biol 35, 295-302.

Morgan, L. W., Greene, A. V. \& Bell-Pedersen, D. (2003). Circadian and light-induced expression of luciferase in Neurospora crassa. Fungal Genet Biol 38, 327-332.

O'Brian, G. R., Fakhoury, A. M. \& Payne, G. A. (2003). Identification of genes differentially expressed during aflatoxin biosynthesis in Aspergillus flavus and Aspergillus parasiticus. Fungal Genet Biol 39, 118-127.

Peterbauer, C. K., Lorito, M., Hayes, C. K., Harman, G. E. \& Kubicek, C. P. (1996). Molecular cloning and expression of the nag1 gene ( $N$-acetyl- $\beta$-D-glucosaminidase-encoding gene) from Trichoderma harzianum P1. Curr Genet 30, 325-331. 
Peterbauer, C. K., Brunner, K., Mach, R. L. \& Kubicek, C. P. (2002). Identification of the $N$-acetyl-D-glucosamine-inducible element in the promoter of the Trichoderma atroviride nag1 gene encoding $\mathrm{N}$ acetyl-glucosaminidase. Mol Genet Genomics 267, 162-170.

Ramot, O., Viterbo, A., Friesem, D., Oppenheim, A. \& Chet, I. (2004). Regulation of two homodimer hexosaminidases in the mycoparasitic fungus Trichoderma asperellum by glucosamine. Curr Genet 45, 205-213.

Reithner, B., Brunner, K., Schuhmacher, R., Peissl, I., Seidl, V., Krska, R. \& Zeilinger, S. (2005). The G protein $\alpha$ subunit Tgal of Trichoderma atroviride is involved in chitinase formation and differential production of antifungal metabolites. Fungal Genet Biol 42, 749-760.

Sanz, L., Montero, M., Redondo, J., Llobell, A. \& Monte, E. (2005). Expression of an $\alpha$-1,3-glucanase during mycoparasitic interaction of Trichoderma asperellum. FEBS J 272, 493-499.

Schmoll, M., Zeilinger, S., Mach, R. L. \& Kubicek, C. P. (2004). Cloning of genes expressed early during cellulase induction in Hypocrea jecorina by a rapid subtraction hybridization approach. Fungal Genet Biol 41, 877-887.

Schoffelmeer, E. A., Klis, F. M., Sietsma, J. H. \& Cornelissen, B. J. (1999). The cell wall of Fusarium oxysporum. Fungal Genet Biol 27, 275-282.

Seidl, V., Seiboth, B., Karaffa, L. \& Kubicek, C. P. (2004). The fungal STRE-element-binding protein Seb1 is involved but not essential for glycerol dehydrogenase ( $g l d 1$ ) gene expression and glycerol accumulation in Trichoderma atroviride during osmotic stress. Fungal Genet Biol 41, 1132-1140.

Seidl, V., Huemer, B., Seiboth, B. \& Kubicek, C. P. (2005). A complete survey of Trichoderma chitinases reveals three distinct subgroups of family 18 chitinases. FEBS J 272, 5923-5939.
Tanzer, M. M., Arst, H. N., Skalchunes, A. R., Coffin, M., Darveaux, B. A., Heiniger, R. W. \& Shuster, J. R. (2003). Global nutritional profiling for mutant and chemical mode-of-action analysis in filamentous fungi. Funct Integr Genomics 3, 160-170.

te Biesebeke, R., Boussier, A., van Biezen, N., van den Hondel, C. A. M. J. J. \& Punt, P. J. (2005a). Identification of secreted proteins of Aspergillus oryzae associated with growth on solid cereal substrates. J Biotechnol 121, 482-485.

te Biesebeke, R., van Biezen, N., de Vos, W. M., van den Hondel, C. A. M. J. J. \& Punt, P. J. (2005b). Different control mechanisms regulate glucoamylase and protease gene transcription in Aspergillus oryzae in solid-state and submerged fermentation. Appl Microbiol Biotechnol 67, 75-82.

Tomazett, P. K., Cruz, A. H., Bonfim, S. M., Soares, C. M. \& Pereira, M. (2005). The cell wall of Paracoccidioides brasiliensis: insights from its transcriptome. Genet Mol Res 4, 309-325.

Viterbo, A., Haran, S., Friesem, D., Ramot, O. \& Chet, I. (2001). Antifungal activity of a novel endochitinase gene (chit36) from Trichoderma harzianum Rifai TM. FEMS Microbiol Lett 200, 169-174.

Viterbo, A., Montero, M., Ramot, O., Friesem, D., Monte, E., Llobell, A. \& Chet, I. (2002). Expression regulation of the endochitinase chit36 from Trichoderma asperellum (T. harzianum T-203). Curr Genet 42, 114-122.

Wolski, E. A., Lima, C., Agusti, R., Daleo, G. R., Andreu, A. B. \& de Lederkremer, R. M. (2005). An $\alpha$-glucan elicitor from the cell wall of a biocontrol binucleate Rhizoctonia isolate. Carbohydr Res 340, 619-627.

Yagi, T., Hisada, R. \& Shibata, H. (1989). 3,4-Dinitrophenyl $N$-acetyl- $\beta$-D-glucosaminide, a synthetic substrate for direct spectrophotometric assay of $N$-acetyl- $\beta$-D-glucosaminidase or $N$-acetyl- $\beta$-Dhexosaminidase. Anal Biochem 183, 245-249. 